\title{
Disturbance Rejection for Fractional-Order Time-Delay Systems
}

\author{
Hai-Peng Jiang and Yong-Qiang Liu \\ School of Electric Power, South China University of Technology, Guangzhou, Guangdong 510641, China \\ Correspondence should be addressed to Hai-Peng Jiang; 2932521062@qq.com \\ Received 8 April 2016; Accepted 10 May 2016 \\ Academic Editor: Riccardo Caponetto
}

Copyright (C) 2016 H.-P. Jiang and Y.-Q. Liu. This is an open access article distributed under the Creative Commons Attribution License, which permits unrestricted use, distribution, and reproduction in any medium, provided the original work is properly cited.

\begin{abstract}
This paper presents an equivalent-input-disturbance (EID-) based disturbance rejection method for fractional-order time-delay systems. First, a modified state observer is applied to reconstruct the state of the fractional-order time-delay plant. Then, a disturbance estimator is designed to actively compensate for the disturbances. Under such a construction of the system, by constructing a novel monochromatic Lyapunov function and using direct Lyapunov approach, the stability analysis and controller design algorithm are derived in terms of linear matrix inequality (LMI) technique. Finally, simulation results demonstrate the validity of the proposed method.
\end{abstract}

\section{Introduction}

Fractional calculus is a generalization of ordinary differentiation and integration to arbitrary (noninteger) order [1]. It was found that the description of some dynamic systems in interdisciplinary fields is more accurate when the fractional derivative is used $[2,3]$. In the meantime, time-delays are inherent in many dynamic systems [4]. The delay terms may degrade the achievable control performance or even cause instability. In recent years, considerable attention has been paid to fractional-order time-delay systems $[5,6]$.

The question of stability is of main interest in the control theory. For integer-order systems, the second method of Lyapunov provides a way to analyze the stability of a system without explicitly solving the differential equations. On the base of this method, Li et al. proposed Lyapunov direct theorem for fractional system [7]. Based on the same idea, Baleanu et al. extended the theorem to fractional systems with delay and propose a fractional Lyapunov-Krasovskii stable theorem [8]. The idea of these fractional Lyapunov theorems is derived by constructing a positive definite function and calculating the fractional derivative of this function. However, by now there is not an effectively approach to deal with it, especially the fractional system with delay.

On the other hand, to enhance the control performance, the disturbance rejection for time-delay systems has been addressed in the control theory and engineering. Balochian et al. proposed a sliding mode control law to handle matched disturbances for the fractional-order systems with state delay [9]. $H_{\infty}$ control is widely used in disturbance rejection of both the integer-order and fractional-order systems. To calculate the $H_{\infty}$ performance, Moze et al. established a bounded real lemma for commensurate fractional-order systems [10]. However, even for the simplest state-feedback stabilization problem, it is not so easy to use parametrization techniques to obtain the feedback gain since the corresponding LMI involves two complex matrix variables. The statefeedback $H_{\infty}$ control problem for fractional-order systems was first discussed by Zhuang and Zhong [11]. Shen and Lam [12] addressed the state- feedback $H_{\infty}$ suboptimal control problem for fractional-order linear systems. By introducing a new real matrix variable, the feedback gain is decoupled with complex matrix variables and further parameterized by the new matrix variable. Due to the time-delay terms and the shortcoming of fractional direct Lyapunov theorem, to our best knowledge, there is no work relating to the $H_{\infty}$ control problem of fractional-order time-delay systems in the existing literature. Fortunately, the equivalent-input-disturbance (EID) $[13,14]$ is another effective approach to reject both matched and unmatched disturbances for integer-order linear systems [15]. 
The main objective of this paper is to extend EID disturbance rejection method to fractional-order time-delay systems. The main contribution of this paper is twofold. First, a modified state observer is used to reconstruct the state of the time-delay system in the EID-based control scheme. Second, by using direct Lyapunov approach, a novel monochromatic Lyapunov function was constructed and a stability condition as well as a design algorithm for the control system is derived using LMI techniques.

The rest of this paper is organized as follows. Preliminaries and problem formulation are provided in Section 2. In Section 3, an LMI-based stability condition and controller design algorithm are presented. A numerical example is illustrated to show the validity and superiority of the proposed method in Section 4, and a conclusion follows in Section 5.

\section{Preliminaries and Problem Formulation}

In this section, some basic definitions and properties (for more details see $[1,2]$ ) are introduced, which will be used in the following sections.

The definitions for fractional derivative commonly used are Grunwald-Letnikov (GL), Riemann-Liouville (RL), and Caputo (C) definition. The advantage of Caputo approach is that the initial conditions for fractional differential equations with Caputo derivative take on the same form as those for integer-order ones [3]. In this paper, we adopt the Caputo definition for fractional derivative.

Definition 1 (Podlubny [1]). The Caputo derivative is defined by

$$
{ }_{t_{0}}^{C} \mathscr{D}_{t}^{\alpha} f(t)=\frac{1}{\Gamma(n-\alpha)} \int_{t_{0}}^{t}(t-\tau)^{-\alpha+n-1} f^{(n)}(\tau) d \tau,
$$

where $\Gamma(\cdot)$ is the well-known Gamma function which is defined by $\Gamma(z)=\int_{0}^{\infty} e^{-z} t^{z-1} d t$ and $n$ is the first integer which is not less than $\alpha$, that is, $\alpha \in[n-1, n)$.

Definition 2 (see [16]). Let $h(t)$ be the impulse response of a linear system. The diffusive representation (or frequency weighting function) of $h(t)$ is called $\mu(\omega)$ with the following relation:

$$
h(t)=\int_{0}^{\infty} \mu(\omega) e^{-\omega t} d \omega
$$

while the diffusive representation of $\mu(\omega)$ is introduced as

$$
\mu(\omega)=\frac{\sin (a \pi)}{\pi} \omega^{-a}
$$

Remark 3 (see [16]). It is worth noting that for the fractionalorder integral operator $t_{0} \mathscr{D}_{t}^{-a} f(t)$ can be written as

$$
{ }_{t_{0}} \mathscr{D}_{t}^{-a} f(t)=h(t) * f(t)
$$

where $*$ denote convolution operator and $h(t)=t^{q-1} / \Gamma(q)$.
Lemma 4 (see [16]). The fractional-order nonlinear differential equation

$$
{ }_{t_{0}}^{C} \mathscr{D}_{t}^{\alpha} x(t)=f(x(t))
$$

due to the continuous frequency distributed model of the fractional integrator can be expressed as

$$
\begin{gathered}
\frac{\partial z(\omega, t)}{\partial t}=-\omega z(\omega, t)+f(x(t)), \\
x(t)=\int_{0}^{\infty} \mu(\omega) z(\omega, t) d \omega,
\end{gathered}
$$

where $\mu(\omega)$ is the same as in Definition 2.

In this paper, we consider the following factional-order time-delay system:

$$
\begin{aligned}
{ }_{t_{0}}^{C} \mathscr{D}_{t}^{\alpha} \mathbf{x}(t) & =\mathbf{A} \mathbf{x}(t)+\mathbf{A}_{d} \mathbf{x}(t-h)+\mathbf{B}(\mathbf{u}(t)+\mathbf{d}(t)), \\
\mathbf{y}(t) & =\mathbf{C} \mathbf{x}(t), \\
\mathbf{x}(t) & =\boldsymbol{\xi}(t), \quad t \in[-h, 0],
\end{aligned}
$$

where $\mathbf{x}(t) \in \mathbb{R}^{n}, \mathbf{u}(t) \in \mathbb{R}^{p}, \mathbf{y}(t) \in \mathbb{R}^{q}$, and $\mathbf{d}(t) \in \mathbb{R}^{p}$ are the state of the plant, the control input, the output, and an external disturbance, respectively. $\mathbf{A}, \mathbf{B}$, and $\mathbf{C}$ are constant matrices of appropriate dimensions. $\boldsymbol{\xi}(t)$ is a given continuous initial state vector, and $h$ is a positive time-delay.

For convenience of discussion, we assume that $\mathbf{C}$ has full row rank and its singular-value decomposition is

$$
\mathbf{C}=\mathbf{U}\left[\begin{array}{ll}
\mathbf{S} & 0
\end{array}\right] \mathbf{V}^{T},
$$

where $\mathbf{S}$ is a semipositive definite matrix and $\mathbf{U}$ and $\mathbf{V}$ are unitary matrices.

The definition of the EID-based disturbance rejection is defined as follows.

Definition 5 (She et al. [13]). For a controlled system, let the input $\mathbf{u}(t)$ be zero. A signal, $\mathbf{d}_{e}(t)$, on the control input channel is called an EID of the disturbance $\mathbf{d}(t)$, if it produces the same effect on the output as the disturbance $\mathbf{d}(t)$ does for all $t \geq 0$.

In the following, we consider the EID-based disturbance rejection for fractional-order time- delay system in Figure 1. The controllable and observable plant with Caputo definition of the fractional-order derivative is described by time-delay system (7).

In Figure 1, the internal model is described by

$$
{ }_{t_{0}}^{C} \mathscr{D}_{t}^{\alpha} \mathbf{x}_{R}(t)=\mathbf{A}_{R} \mathbf{x}_{R}(t)+\mathbf{B}_{R}[\mathbf{r}(t)-\mathbf{y}(t)]
$$

which is used to guarantee perfect tracking error for a known reference input, $\mathbf{r}(t)$. Since the reference input is known, $\mathbf{A}_{R}$ and $\mathbf{B}_{R}$ can be determined directly [15]. For example, if the reference input is a step signal, the parameters of the internal model could be selected as $\mathbf{A}_{R}=0$ and $\mathbf{B}_{R}=1$. 


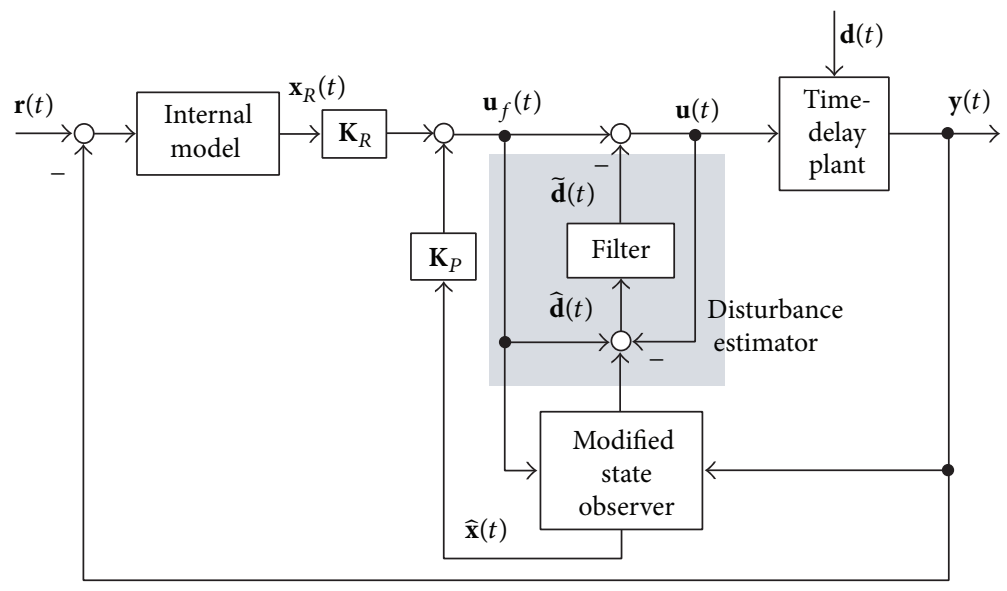

FIGURE 1: Configuration of the EID-based time-delay system.

To reproduce the state of the time-delay plant (7), we choose the following modified full-order state observer [17]:

$$
\begin{aligned}
{ }_{t_{0}}^{C} \mathscr{D}_{t}^{\alpha} \widehat{\mathbf{x}}(t)= & \widehat{\mathbf{A}} \widehat{\mathbf{x}}(t)+\mathbf{A}_{d} \widehat{\mathbf{x}}(t-h)+\mathbf{L}[\mathbf{y}(t)-\mathbf{C} \widehat{\mathbf{x}}(t)] \\
& +\mathbf{B} \mathbf{u}_{f}(t), \\
\widehat{\mathbf{y}}(t)= & \mathbf{C} \widehat{\mathbf{x}}(t),
\end{aligned}
$$

where $\widehat{\mathbf{x}}(t)$ is the reconstruction state of $\mathbf{x}(t)$ and the matrices $\widehat{\mathbf{A}}$ and $\mathbf{L}$ are to be determined.

Remark 6. Unlike the Luenberger-type observer constructed in $[14,15]$, fractional-order time-delay observer (10) contains two unknown system matrices, $\widehat{\mathbf{A}}$ and $\mathbf{L}$, which give an opportunity to better adjust the dynamical stability of the observer-based fractional-order control system [17].

The state-feedback control law $\mathbf{u}_{f}(t)$ in Figure 1 is designed to be

$$
\mathbf{u}_{f}(t)=\mathbf{K}_{R} \mathbf{x}_{R}(t)+\mathbf{K}_{P} \widehat{\mathbf{x}}(t),
$$

where $\mathbf{K}_{R}$ and $\mathbf{K}_{P}$ are the gain matrices to be designed.

As discussed in [13], the estimation of the EID $\widehat{\mathbf{d}}(t)$ in Figure 1 is designed as

$$
\widehat{\mathbf{d}}(t)=\mathbf{B}^{+} \mathbf{L}[\mathbf{y}(t)-\widehat{\mathbf{y}}(t)]+\mathbf{u}_{f}(t)-\mathbf{u}(t),
$$

where $\mathbf{B}^{+}=\left(\mathbf{B}^{T} \mathbf{B}\right)^{-1} \mathbf{B}^{T}$. In fact, letting $\Delta \mathbf{x}(t)=\widehat{\mathbf{x}}(t)-\mathbf{x}(t)$ and substituting it into fractional-order time-delay system (7) yield

$$
\begin{aligned}
{ }_{t_{0}}^{C} \mathscr{D}_{t}^{\alpha} \widehat{\mathbf{x}}(t)= & \widehat{\mathbf{A}} \widehat{\mathbf{x}}(t)+\mathbf{A}_{d} \widehat{\mathbf{x}}(t-h)+\mathbf{B u}(t)+\mathbf{B d}(t) \\
& +\Delta \dot{\mathbf{x}}(t)-\widehat{\mathbf{A}} \Delta \mathbf{x}(t)-\mathbf{A}_{d} \Delta \mathbf{x}(t-h) \\
& -(\widehat{\mathbf{A}}-\mathbf{A}) \mathbf{x}(t)
\end{aligned}
$$

Assume that there exists a control input $\Delta \mathbf{d}(t)$ that satisfies

$$
\begin{aligned}
\mathbf{B} \Delta \mathbf{d}(t)= & \Delta \dot{\mathbf{x}}(t)-\widehat{\mathbf{A}} \Delta \mathbf{x}(t)-\mathbf{A}_{d} \Delta \mathbf{x}(t-h) \\
& -(\widehat{\mathbf{A}}-\mathbf{A}) \mathbf{x}(t) .
\end{aligned}
$$

Substituting (14) into (13) and letting the estimate of the EID be

$$
\widehat{\mathbf{d}}(t)=\mathbf{d}(t)+\Delta \mathbf{d}(t)
$$

allow us to express observer (10) as

$$
{ }_{t_{0}}^{C} \mathscr{D}_{t}^{\alpha} \widehat{\mathbf{x}}(t)=\widehat{\mathbf{A}} \widehat{\mathbf{x}}(t)+\mathbf{A}_{d} \widehat{\mathbf{x}}(t-h)+\mathbf{B u}(t)+\mathbf{B} \widehat{\mathbf{d}}(t) .
$$

Observer (10) and (16) yield

$$
\mathbf{B}\left[\widehat{\mathbf{d}}(t)+\mathbf{u}(t)-\mathbf{u}_{f}(t)\right]=\mathbf{L}[\mathbf{y}(t)-\widehat{\mathbf{y}}(t)] .
$$

If we solve (17) for $\widehat{\mathbf{d}}(t)$, then a least square solution is (12).

Since the output, $\mathbf{y}(t)$, contains measurement noise, a lowpass filter is used to select the angular frequency band for the disturbance estimate. The state-space form of the filter is described as

$$
\begin{aligned}
{ }_{t_{0}}^{C} \mathscr{D}_{t}^{\alpha} \mathbf{x}_{\mathbf{F}}(t) & =\mathbf{A}_{\mathbf{F}} \mathbf{x}_{\mathbf{F}}(t)+\mathbf{B}_{\mathbf{F}} \widehat{\mathbf{d}}(t), \\
\widetilde{\mathbf{d}}(t) & =\mathbf{C}_{\mathbf{F}} \mathbf{x}_{\mathbf{F}}(t),
\end{aligned}
$$

where $\mathbf{x}_{\mathbf{F}}(t)$ is the state of the filter and $\widetilde{\mathbf{d}}(t)$ is the filtered signal. The transfer function of the filter $\mathbf{F}(s)$ satisfies

$$
|\mathbf{F}(j \omega)| \approx 1, \quad \forall \omega \in\left[0, \omega_{r}\right],
$$

where $\omega_{r}$ is the highest angular frequency selected for disturbance estimation. A suitable filter has its cutoff angular frequency being more than 10 times larger than $\omega_{r}$.

The purpose of this paper is to investigate the design problem of the full-order state observer (10) and the EIDbased state-feedback control law $\mathbf{u}(t)$ :

$$
\mathbf{u}(t)=\mathbf{u}_{f}(t)-\widetilde{\mathbf{d}}(t) .
$$

\section{Analysis and Controller Design of the Closed-Loop System}

In this section, we will present an LMI-based method for both stability analysis and the parameter design for the proposed control law. 
First, set the exogenous signals to be zero, that is,

$$
\begin{gathered}
\mathbf{r}(t)=0 \\
\mathbf{d}(t)=0 .
\end{gathered}
$$

Then the fractional-order time-delay system (7) becomes

$$
\begin{aligned}
{ }_{t_{0}}^{C} \mathscr{D}_{t}^{\alpha} \mathbf{x}(t) & =\mathbf{A} \mathbf{x}(t)+\mathbf{A}_{d} \mathbf{x}(t-h)+\mathbf{B u}(t), \\
\mathbf{y}(t) & =\mathbf{C} \mathbf{x}(t) .
\end{aligned}
$$

Define

$$
\Delta \mathbf{x}(t)=\mathbf{x}(t)-\widehat{\mathbf{x}}(t) .
$$

It follows from (23) and (10) that

$$
\begin{aligned}
{ }_{t_{0}}^{C} \mathscr{D}_{t}^{\alpha} \widehat{\mathbf{x}}(t)= & \widehat{\mathbf{A}} \widehat{\mathbf{x}}(t)+\mathbf{L C} \Delta \mathbf{x}(t)+\mathbf{B} \mathbf{u}_{f}(t) \\
& +\mathbf{A}_{d} \widehat{\mathbf{x}}(t-h) .
\end{aligned}
$$

From (10), (18), (20), (22), and (23), we have

$$
\begin{aligned}
{ }_{t_{0}}^{C} \mathscr{D}_{t}^{\alpha}(\Delta \mathbf{x}(t))= & (\mathbf{A}-\mathbf{L C}) \Delta \mathbf{x}(t)-\mathbf{B C}_{\mathbf{F}} \mathbf{x}_{\mathbf{F}}(t) \\
& +\mathbf{A}_{d} \Delta \mathbf{x}(t-h)+(\mathbf{A}-\widehat{\mathbf{A}}) \widehat{\mathbf{x}}(t) .
\end{aligned}
$$

Combining (12) and (20), filter (18) becomes

$$
{ }_{t_{0}}^{C} \mathscr{D}_{t}^{\alpha} \mathbf{x}_{\mathbf{F}}(t)=\mathbf{B}_{\mathbf{F}} \mathbf{B}^{+} \mathbf{L} \mathbf{C} \Delta \mathbf{x}(t)+\left(\mathbf{A}_{\mathbf{F}}+\mathbf{B}_{\mathbf{F}} \mathbf{C}_{\mathbf{F}}\right) \mathbf{x}_{\mathbf{F}}(t) .
$$

Substituting (21) and (23) into (9) yields the following form of the internal model:

$$
{ }_{t_{0}}^{C} \mathscr{D}_{t}^{\alpha} \mathbf{x}_{R}(t)=-\mathbf{B}_{R} \mathbf{C} \widehat{\mathbf{x}}(t)-\mathbf{B}_{R} \mathbf{C} \Delta \mathbf{x}(t)+\mathbf{A}_{R} \mathbf{x}_{R}(t) .
$$

Set

$$
\boldsymbol{\varphi}(t)=\left[\begin{array}{llll}
\widehat{\mathbf{x}}^{T}(t) & \Delta \mathbf{x}^{T}(t) & \mathbf{x}_{F}^{T}(t) & \mathbf{x}_{R}^{T}(t)
\end{array}\right]^{T}
$$

Then the state-space representation of the closed-loop system in Figure 1 is derived as follows:

$$
{ }_{t_{0}}^{C} \mathscr{D}_{t}^{\alpha} \boldsymbol{\varphi}(t)=\overline{\mathbf{A}} \boldsymbol{\varphi}(t)+\overline{\mathbf{A}}_{d} \boldsymbol{\varphi}(t-h)+\overline{\mathbf{B}} \mathbf{u}_{f}(t),
$$

where

$$
\begin{aligned}
\overline{\mathbf{A}} & =\left[\begin{array}{cccc}
\widehat{\mathbf{A}} & \mathbf{L C} & \mathbf{0} & \mathbf{0} \\
\mathbf{A}-\widehat{\mathbf{A}} & \mathbf{A}-\mathbf{L C} & -\mathbf{B C}_{F} & \mathbf{0} \\
\mathbf{0} & \mathbf{B}_{F} \mathbf{B}^{+} \mathbf{L C} & \mathbf{A}_{F}+\mathbf{B}_{F} \mathbf{C}_{F} & \mathbf{0} \\
-\mathbf{B}_{R} \mathbf{C} & -\mathbf{B}_{R} \mathbf{C} & \mathbf{0} & \mathbf{A}_{R}
\end{array}\right], \\
\overline{\mathbf{B}} & =\left[\begin{array}{llll}
\mathbf{B}^{T} & \mathbf{0} & \mathbf{0} & \mathbf{0}
\end{array}\right]^{T}, \\
\overline{\mathbf{A}}_{d} & =\left[\begin{array}{cccc}
\mathbf{A}_{d} & \mathbf{0} & \mathbf{0} & \mathbf{0} \\
\mathbf{0} & \mathbf{A} & 0 & 0 \\
\mathbf{0} & \mathbf{0} & \mathbf{0} & \mathbf{0} \\
\mathbf{0} & \mathbf{0} & \mathbf{0} & \mathbf{0}
\end{array}\right] .
\end{aligned}
$$

The state-feedback control law (11) is expressed as

$$
\mathbf{u}_{f}(\mathbf{t})=\overline{\mathbf{K}} \varphi(\mathbf{t})
$$

where

$$
\overline{\mathbf{K}}=\left[\begin{array}{llll}
\mathbf{K}_{P} & \mathbf{0} & \mathbf{0} & \mathbf{K}_{R}
\end{array}\right] .
$$

Substituting (31) into (29), we obtain the following general form of time-delay systems:

$$
{ }_{t_{0}}^{C} \mathscr{D}_{t}^{\alpha} \boldsymbol{\varphi}(t)=\widehat{\overline{\mathbf{A}}} \boldsymbol{\varphi}(t)+\overline{\mathbf{A}}_{d} \boldsymbol{\varphi}(t-h),
$$

where

$$
\widehat{\overline{\mathbf{A}}}=\left[\begin{array}{cccc}
\widehat{\mathbf{A}}+\mathbf{B K}_{P} & \mathbf{L C} & \mathbf{0} & \mathbf{B} \mathbf{K}_{R} \\
\mathbf{A}-\widehat{\mathbf{A}} & \mathbf{A}-\mathbf{L C} & -\mathbf{B C}_{F} & \mathbf{0} \\
0 & \mathbf{B}_{F} \mathbf{B}^{+} \mathbf{L C} & \mathbf{A}_{F}+\mathbf{B}_{F} \mathbf{C}_{F} & \mathbf{0} \\
-\mathbf{B}_{R} \mathbf{C} & -\mathbf{B}_{R} \mathbf{C} & \mathbf{0} & \mathbf{A}_{R}
\end{array}\right] .
$$

Now, the design problem can be stated as a stabilization problem of fractional-order time-delay system (33).

Remark 7. As we all know, plenty of LMI-based stability and stabilization conditions have been proposed for integer-order time-delay systems. However, by now, there is not a simple and effective approach to stabilize a fractional-order timedelay system according to the direct Lyapunov theorem and LMI technique.

In the following, we will propose a Lyapunov theorem for fractional-order system with delay and get an LMI-based stability condition.

Lemma 8. The fractional-order time-delay system (33) is asymptotically stable, if there exist symmetric positive definite matrices $\mathbf{P}$ and $\mathbf{Q}$, such that the following LMI is feasible:

$$
\left[\begin{array}{cc}
\mathbf{P} \overline{\mathbf{A}}+\widehat{\overline{\mathbf{A}}}^{T} \mathbf{P}+\mathbf{Q} & \mathbf{P} \overline{\mathbf{A}}_{d} \\
* & -\mathbf{Q}
\end{array}\right]<\mathbf{0} .
$$

Proof. It follows from Lemma 4 that the fractional-order time-delay system (33) can be written as

$$
\begin{gathered}
\frac{\partial \mathbf{Z}(\omega, t)}{\partial t}=-\omega \mathbf{Z}(\omega, t)+\widehat{\overline{\mathbf{A}}} \varphi(t)+\overline{\mathbf{A}}_{d} \boldsymbol{\varphi}(t-h), \\
\varphi(t)=\int_{0}^{\infty} \mu(\omega) \mathbf{Z}(\omega, t) d \omega .
\end{gathered}
$$

Let us consider the Lyapunov functions: $v(\omega, t)=$ $\mathbf{Z}^{T}(\omega, t) \mathbf{P Z}(\omega, t)$ is the monochromatic Lyapunov function corresponding to the elementary frequency $\omega$ and $V_{1}(t)$ is the Lyapunov function integrating all the monochromatic $v(\omega, t)$ with the weighting function $\mu(\omega)$ on the whole spectral range [18]. That is to say, we define our monochromatic Lyapunov function as

$$
\begin{aligned}
V_{1}(t) & =\int_{0}^{\infty} \mu(\omega) v(\omega, t) d \omega \\
& =\int_{0}^{\infty} \mu(\omega) \mathbf{Z}^{T}(\omega, t) \mathbf{P Z}(\omega, t) d \omega,
\end{aligned}
$$

where $\mathbf{P} \in \mathbb{R}^{n \times n}$ is symmetric and positive definite. 
The time derivative of $V_{1}(t)$ taken along the solution trajectories of (36) is

$$
\begin{aligned}
& \dot{V}_{1}(t)=\int_{0}^{\infty} \mu(\omega) \\
& \cdot\left\{-\omega \mathbf{Z}^{T}(\omega, t)+\boldsymbol{\varphi}^{T}(t) \widehat{\widehat{\mathbf{A}}}^{T}+\varphi^{T}(t-h) \overline{\mathbf{A}}_{d}^{T}\right\} \\
& \cdot \mathbf{P Z}(\omega, t) d \omega+\int_{0}^{\infty} \mu(\omega) \mathbf{Z}^{T}(\omega, t) \\
& \cdot \mathbf{P}\left\{-\omega \mathbf{Z}(\omega, t)+\widehat{\overline{\mathbf{A}}} \boldsymbol{\varphi}(t)+\overline{\mathbf{A}}_{d} \boldsymbol{\varphi}(t-h)\right\} d \omega \\
& =-2 \int_{0}^{\infty} \omega \mu(\omega) \mathbf{Z}^{T}(\omega, t) \mathbf{P Z}(\omega, t) d \omega \\
& +2 \boldsymbol{\varphi}^{T}(t) \mathbf{P} \widehat{\overline{\mathbf{A}}} \boldsymbol{\varphi}(t)+2 \boldsymbol{\varphi}^{T}(t) \mathbf{P} \overline{\mathbf{A}}_{d} \boldsymbol{\varphi}(t-h) .
\end{aligned}
$$

Now, a second Lyapunov-Krasovskii function candidate $V_{2}(t)$ is defined by

$$
V_{2}(t)=\int_{t-h}^{t} \boldsymbol{\varphi}^{T}(s) \mathbf{Q} \boldsymbol{\varphi}(s) d s,
$$

where $\mathbf{Q} \in \mathbb{R}^{n \times n}$ is a symmetric positive definite matrix. Clearly, the time derivative of $V_{2}(t)$ along the solution of system (33) is given by

$$
\dot{V}_{2}(t)=\boldsymbol{\varphi}^{T}(t) \mathbf{Q} \boldsymbol{\varphi}(t)-\boldsymbol{\varphi}^{T}(t-h) \mathbf{Q} \boldsymbol{\varphi}(t-h) .
$$

Summing the two time derivatives of the Lyapunov functions $V_{1}(t)$ and $V_{2}(t)$, that is, $V(t)=V_{1}(t)+V_{2}(t)$, yields

$$
\begin{aligned}
\dot{V}(t)= & -2 \int_{0}^{\infty} \omega \mu(\omega) \mathbf{Z}^{T}(\omega, t) \mathbf{P Z}(\omega, t) d \omega \\
& +2 \boldsymbol{\varphi}^{T}(t) \mathbf{P} \widehat{\overline{\mathbf{A}}} \boldsymbol{\varphi}(t)+2 \boldsymbol{\varphi}^{T}(t) \mathbf{P} \overline{\mathbf{A}}_{d} \boldsymbol{\varphi}(t-h) \\
& +\boldsymbol{\varphi}^{T}(t) \mathbf{Q} \boldsymbol{\varphi}(t)-\boldsymbol{\varphi}^{T}(t-h) \mathbf{Q} \boldsymbol{\varphi}(t-h)
\end{aligned}
$$

As a result, we obtain the overall sufficient condition for the stability of system (36) in the following form:

$$
\begin{aligned}
& \boldsymbol{\varphi}(t)^{T}\left[\mathbf{P} \widehat{\overline{\mathbf{A}}}+\widehat{\overline{\mathbf{A}}}^{T} \mathbf{P}+\mathbf{Q}\right] \boldsymbol{\varphi}(t)-\boldsymbol{\varphi}^{T}(t-h) \mathbf{Q} \boldsymbol{\varphi}(t-h) \\
& \quad+\boldsymbol{\varphi}(t)^{T} \mathbf{P} \overline{\mathbf{A}}_{d} \boldsymbol{\varphi}(t-h)+\boldsymbol{\varphi}^{T}(t-h) \overline{\mathbf{A}}_{d} \mathbf{P} \boldsymbol{\varphi}(t) \\
& \quad \leq 0,
\end{aligned}
$$

which is equivalent to

$$
\mathbf{\Omega}^{T}\left[\begin{array}{cc}
\mathbf{P} \widehat{\overline{\mathbf{A}}}+\widehat{\overline{\mathbf{A}}}^{T} \mathbf{P}+\mathbf{Q} & \mathbf{P} \overline{\mathbf{A}}_{d} \\
* & -\mathbf{Q}
\end{array}\right] \mathbf{\Omega} \leq \mathbf{0},
$$

where $\boldsymbol{\Omega}=\left[\begin{array}{c}\boldsymbol{\varphi}(t)^{T} \\ \varphi^{T}(t-\tau)\end{array}\right]$.

Therefore, if

$$
\left[\begin{array}{cc}
\mathbf{P} \overline{\overline{\mathbf{A}}}+\widehat{\overline{\mathbf{A}}}^{T} \mathbf{P}+\mathbf{Q} & \mathbf{P} \overline{\mathbf{A}}_{d} \\
* & -\mathbf{Q}
\end{array}\right]<\mathbf{0},
$$

then, the fractional-order time-delay system (33) is asymptotically stable. The proof is completed.
Remark 9. By constructing a novel monochromatic Lyapunov function and using direct Lyapunov approach, a sufficient condition of stability for fractional-order time-delay systems is obtained. It is worth mentioning that the authors in [19] have also proposed a direct Lyapunov stable theorem for fractional-order time-delay system (33). However, as pointed out in [20], their proof is not correct.

Remark 10. Based on the proposed stability condition, we can easily analyze the stability of a fractional-order timedelay system and design a stabilization controller to stabilize a fractional system with delay by many approaches which have been used in integer-order systems.

To obtain the main result in this paper, we need the following lemmas.

Lemma 11 (Ho and Lu [21]). For a given matrix $\Pi \in \mathbb{R}^{p \times n}$ with rank rank $(\boldsymbol{\Pi})=p$, there exists a matrix $\overline{\mathbf{X}} \in \mathbb{R}^{p \times p}$ such that $\mathbf{\Pi \mathbf { X }}=\overline{\mathbf{X}} \boldsymbol{\Pi}$ holds for any $\mathbf{X} \in \mathbb{R}^{n \times n}$ if and only if $\mathbf{X}$ can be decomposed as

$$
\mathbf{X}=\mathbf{W} \overline{\mathbf{X}} \mathbf{W}^{T}, \quad \overline{\mathbf{X}}=\operatorname{diag}\left\{\overline{\mathbf{X}}_{11}, \overline{\mathbf{X}}_{22}\right\},
$$

where $\mathbf{W} \in \mathbb{R}^{n \times n}$ is a unitary matrix, $\overline{\mathbf{X}}_{11} \in \mathbb{R}^{p \times p}$, and $\overline{\mathbf{X}}_{22} \in$ $\mathbb{R}^{(n-p) \times(n-p)}$.

Lemma 12 (Schur complement, Khargonekar et al. [22]). For a given symmetric matrix

$$
\Sigma=\left[\begin{array}{ll}
\Sigma_{11} & \Sigma_{12} \\
\Sigma_{12}^{T} & \Sigma_{22}
\end{array}\right]
$$

the following statements are equivalent:

(1) $\Sigma<0$,

(2) $\Sigma_{11}<0$ and $\Sigma_{22}-\Sigma_{12}^{T} \Sigma_{11}^{-1} \Sigma_{12}<0$,

(3) $\Sigma_{22}<0$ and $\Sigma_{11}-\Sigma_{12} \Sigma_{22}^{-1} \Sigma_{12}^{T}<0$.

Based on the above lemmas, a stabilization result is established for the EID-based disturbance rejection control systems as follows.

Theorem 13. The fractional-order time-delay system (29) is asymptotically stable under control law (31), if there exist symmetric positive definite matrices $\mathbf{X}_{1}, \mathbf{X}_{11}, \mathbf{X}_{22}, \mathbf{X}_{3}, \mathbf{X}_{4}, \mathbf{Y}_{1}$, $\mathbf{Y}_{2}, \mathbf{Y}_{3}$, and $\mathbf{Y}_{4}$ and appropriate matrices $\mathbf{W}_{1}, \mathbf{W}_{2}$, and $\mathbf{W}_{3}$ such that the following LMI is feasible:

$$
\left[\begin{array}{ccc}
\Phi & \Psi & \mathrm{X} \\
* & -\mathrm{Y} & \mathbf{0} \\
* & * & -\mathrm{Y}
\end{array}\right]<\mathbf{0}
$$


where

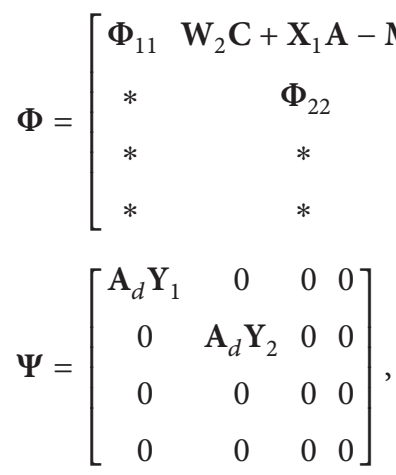

$$
\begin{aligned}
& \mathbf{X}=\operatorname{diag}\left\{\mathbf{X}_{1}, \mathbf{X}_{2}, \mathbf{X}_{3}, \mathbf{X}_{4}\right\} \text {, } \\
& \mathbf{Y}=\operatorname{diag}\left\{\mathbf{Y}_{1}, \mathbf{Y}_{2}, \mathbf{Y}_{3}, \mathbf{Y}_{4}\right\} \text {, } \\
& \mathbf{X}_{2}=\mathbf{V} \operatorname{diag}\left\{\mathbf{X}_{11}, \mathbf{X}_{22}\right\} \mathbf{V}^{T} \text {, } \\
& \boldsymbol{\Phi}_{11}=\mathbf{M}+\mathbf{M}^{T}+\mathbf{X}_{1} \mathbf{A}^{T}+\mathbf{B} \mathbf{W}_{1}+\mathbf{W}_{1}^{T} \mathbf{B}^{T}, \\
& \Phi_{14}=\mathbf{B W}_{3}-\mathbf{X}_{1} \mathbf{C}^{T} \mathbf{B}_{R}^{T}, \\
& \boldsymbol{\Phi}_{22}=\mathbf{A X}_{2}+\mathbf{X}_{2} \mathbf{A}^{T}-\mathbf{W}_{2} \mathbf{C}-\mathbf{C}^{T} \mathbf{W}_{2}^{T}, \\
& \boldsymbol{\Phi}_{23}=-\mathbf{B} \mathbf{C}_{F} \mathbf{X}_{3}+\mathbf{C}^{T} \mathbf{W}_{2}^{T}\left(\mathbf{B}^{+}\right)^{T} \mathbf{B}_{F}^{T}, \\
& \boldsymbol{\Phi}_{33}=\left(\mathbf{A}_{F}+\mathbf{B}_{F} \mathbf{C}_{F}\right) \mathbf{X}_{3}+\mathbf{X}_{3}\left(\mathbf{A}_{F}+\mathbf{B}_{F} \mathbf{C}_{F}\right)^{T}, \\
& \Phi_{44}=\mathbf{A}_{R} \mathbf{X}_{4}+\mathbf{X}_{4} \mathbf{A}_{R}^{T} .
\end{aligned}
$$

Moreover, the gains of the state-feedback controller and the observer are given as

$$
\begin{aligned}
\widehat{\mathbf{A}} & =\mathbf{M} \mathbf{X}_{1}^{-1}, \\
\mathbf{K}_{P} & =\mathbf{W}_{1} \mathbf{X}_{1}^{-1}, \\
\mathbf{K}_{R} & =\mathbf{W}_{3} \mathbf{X}_{4}^{-1}, \\
\mathbf{L} & =\mathbf{W}_{2} \mathbf{U S X}_{11}^{-1} \mathbf{S}^{-1} \mathbf{U}^{-1},
\end{aligned}
$$

where $\mathbf{V}$ and $\mathbf{U}$ are derived from (8).

Proof. Applying Lemma 11, we know that there exists a matrix $\overline{\mathbf{X}}_{2}$, such that

$$
\mathrm{CX}_{2}=\overline{\mathrm{X}}_{2} \mathrm{C}
$$

holds. Substituting (8) into (50) yields

$$
\mathbf{X}_{2}=\mathbf{U S} \overline{\mathbf{X}}_{11} \mathbf{S}^{-1} \mathbf{U}^{T} \text {. }
$$

In terms of Lemma 12, (44) is equivalent to

$$
\boldsymbol{\Theta}=\left[\begin{array}{ccc}
\mathbf{P} \widehat{\overline{\mathbf{A}}}+\widehat{\overline{\mathbf{A}}}^{T} \mathbf{P} & \mathbf{P} \overline{\mathbf{A}}_{d} & \mathbf{Q} \\
* & -\mathbf{Q} & \mathbf{0} \\
* & * & -\mathbf{Q}
\end{array}\right]<0,
$$

in which $\mathbf{P}$ and $\mathbf{Q}$ are supposed to be $\mathbf{P}=$ $\operatorname{diag}\left\{\begin{array}{lllll}\mathbf{P}_{1} & \mathbf{P}_{2} & \mathbf{P}_{3} & \mathbf{P}_{4}\end{array}\right\}$ and $\mathbf{Q}=\operatorname{diag}\left\{\begin{array}{llll}\mathbf{Q}_{1} & \mathbf{Q}_{2} & \mathbf{Q}_{3} & \mathbf{Q}_{4}\end{array}\right\}$, respectively, where $\mathbf{P}_{1}, \mathbf{P}_{2}, \mathbf{P}_{3}$, and $\mathbf{P}_{4}, \mathbf{Q}_{1}, \mathbf{Q}_{2}, \mathbf{Q}_{3}$, and $\mathbf{Q}_{4}$ are symmetric positive definite matrices to be determined.

Let

$$
\begin{array}{r}
\mathbf{P}_{i}^{-1}=\mathbf{X}_{i}, \\
\mathbf{Q}_{i}^{-1}=\mathbf{Y}_{i},
\end{array}
$$

$$
i=1,2,3,4,
$$

$$
\begin{aligned}
& \mathbf{X}=\operatorname{diag}\left\{\mathbf{X}_{1}, \mathbf{X}_{2}, \mathbf{X}_{3}, \mathbf{X}_{4}\right\}, \\
& \mathbf{Y}=\operatorname{diag}\left\{\mathbf{Y}_{1}, \mathbf{Y}_{2}, \mathbf{Y}_{3}, \mathbf{Y}_{4}\right\} .
\end{aligned}
$$

Pre- and postmultiplying (52) by the matrix

$$
\Pi=\operatorname{diag}\{\mathbf{X}, \mathbf{Y}, \mathbf{Y}\},
$$

and combining (51), LMI (47) is obtained. Then, letting

$$
\begin{aligned}
\mathbf{K}_{P} \mathbf{X}_{1} & =\mathbf{W}_{1}, \\
\mathbf{K}_{R} \mathbf{X}_{4} & =\mathbf{W}_{3}, \\
\mathbf{L} \overline{\mathbf{X}}_{2} & =\mathbf{W}_{2}
\end{aligned}
$$

and combining (51) yield (49). The proof is completed.

Using Theorem 13, the parameters of the controller can easily be solved. The design process of the EID-based controller is given as follows.

Algorithm 14.

Step 1. Choose $\mathbf{A}_{R}$ and $\mathbf{B}_{R}$ in the internal model (9) for a known reference input.

Step 2. Choose $\mathbf{A}_{F}, \mathbf{B}_{F}$, and $\mathbf{C}_{F}$ for the low-pass filter (18) such that (19) holds.

Step 3. Find a feasible solution to LMI (47) and calculate $\widehat{\mathbf{A}}$, $\mathbf{K}_{P}, \mathbf{K}_{R}$, and $\mathbf{L}$ from (49).

\section{Numerical Example}

This section presents a numerical example to demonstrate the validity of the proposed design method.

Assume that the parameters of plant (7) are

$$
\begin{aligned}
\mathbf{A} & =\left[\begin{array}{cc}
-2 & 3 \\
4 & -5
\end{array}\right], \\
\mathbf{A}_{d} & =\left[\begin{array}{ll}
0.3 & 0 \\
0.1 & 0.4
\end{array}\right], \\
\mathbf{B} & =\left[\begin{array}{l}
1 \\
2
\end{array}\right],
\end{aligned}
$$




$$
\begin{aligned}
\mathbf{C} & =\left[\begin{array}{ll}
5 & 0
\end{array}\right], \\
h & =2, \\
\alpha & =0.90 .
\end{aligned}
$$

A unit step signal is introduced as the reference input at $t=0$ and the following external disturbance

$$
\mathbf{d}(t)=-0.5
$$

is added to the plant at $t=10 \mathrm{~s}$.

In terms of Algorithm 14, first, the parameters of the internal model (9) were selected as

$$
\begin{aligned}
& \mathbf{A}_{R}=-0.001, \\
& \mathbf{B}_{R}=1
\end{aligned}
$$

to track the reference input, $\mathbf{r}(t)$. Since the reference input is a step signal, the parameters of the internal model should be $\mathbf{A}_{R}=0, \mathbf{B}_{R}=1$. Here we chose -0.001 for parameter $\mathbf{A}_{R}$ instead of 0 in order to guarantee the feasibility of LMI (47). This is reasonable because it would only lead to a very small steady-state tracking error for the control system [15].

A low-pass filter is selected as

$$
F(s)=\frac{100}{s^{\alpha}+101}
$$

to meet the condition in (19). So the parameters of the statespace form of the filter are

$$
\begin{aligned}
& \mathbf{A}_{F}=-101, \\
& \mathbf{B}_{F}=100, \\
& \mathbf{C}_{F}=1 .
\end{aligned}
$$

By Theorem 13 and using MATLAB toolbox, a feasible solution for LMI (47) is obtained as follows:

$$
\begin{aligned}
\widehat{\mathbf{A}} & =\left[\begin{array}{rr}
-466.2215 & 114.9612 \\
228.4710 & -57.9257
\end{array}\right], \\
\mathbf{K}_{P} & =\left[\begin{array}{ll}
-18.5560 & -1.7798
\end{array}\right], \\
\mathbf{K}_{R} & =11.1920, \\
\mathbf{L} & =\left[\begin{array}{ll}
677.1735 & -322.2363
\end{array}\right]^{T} .
\end{aligned}
$$

To simulate the fractional-order systems, an Oustaloup's recursive poles/zeros filter [23] (an integer-order system) of order three is used to approximate the fractional operator $1 / s^{0.9}$, which has an error about $2 \mathrm{~dB}$ in the frequency range $\omega=10^{-2} \mathrm{rad} / \mathrm{s}$ to $\omega=10^{2} \mathrm{rad} / \mathrm{s}$.

The system response without control input is shown in Figure 2, which shows that it is not convergent. However, under control law (31) with the parameters in (61), the system response is asymptotically stable and can track the reference input perfectly even in the presence of external disturbances (see the simulation result in Figure 3).

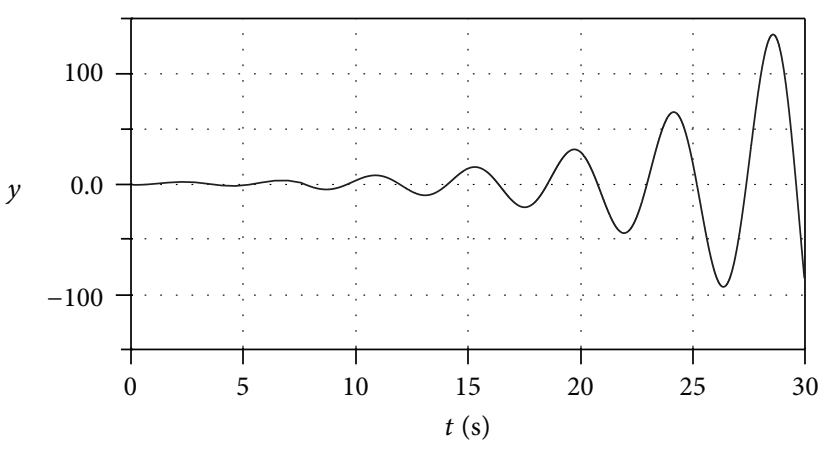

FIGURE 2: System response without control input.

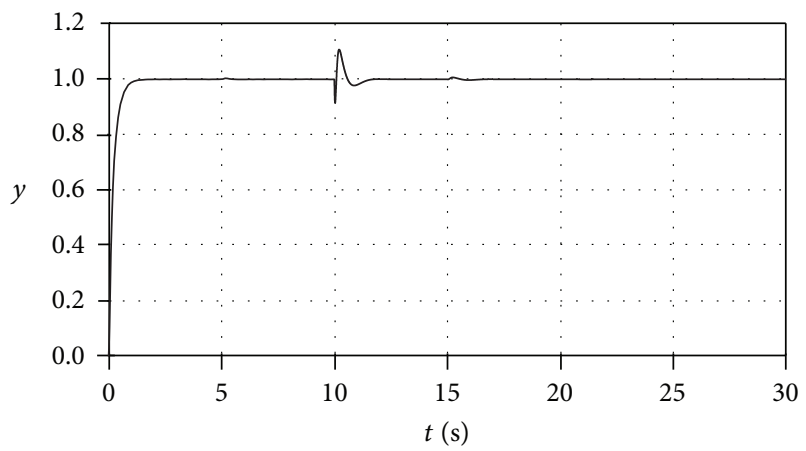

FIGURE 3: System response with the proposed method.

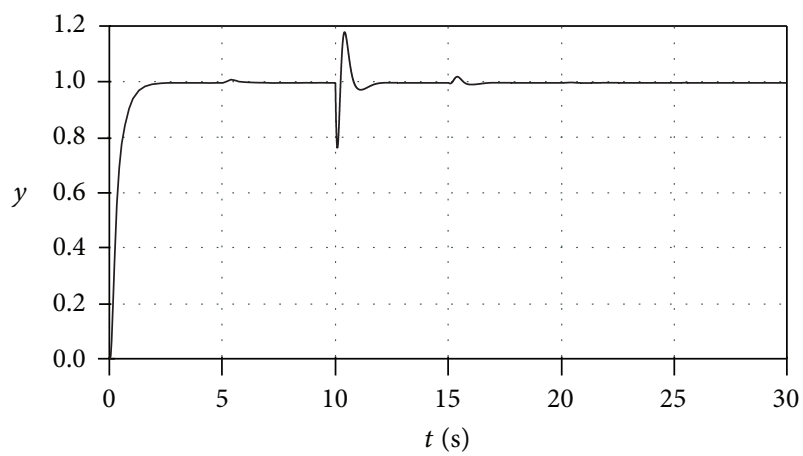

FIGURE 4: System response with the proposed method in [9].

For comparison, we used the proposed method in [9] to carry out simulation. The simulation result is shown in Figure 4. It can be seen from Figures 3 and 4 that our method obtains a better disturbance rejection effect than the proposed method in [9] while these two methods exhibit the same control performance for the reference input.

\section{Conclusion}

This paper has presented an EID-based disturbance rejection method for fractional-order time-delay systems. The configuration with a modified state observer and a disturbance estimator was first constructed. Then, by introducing a continuous frequency distributed equivalent model and using direct Lyapunov approach, the sufficient condition for 
asymptotic stability of the closed-loop fractional-order timedelay system is presented. Based on this stability condition, the parameters of the controller for the closed-loop system can be easily obtained by many approaches which have been used in integer-order systems. Finally, the simulation results demonstrated that the proposed method can reject matched disturbance of fractional-order time-delay systems effectively without knowing any prior information about the disturbances.

\section{Competing Interests}

The authors declare that they have no competing interests.

\section{References}

[1] I. Podlubny, Fractional Differential Equations, Academic Press, New York, NY, USA, 1999.

[2] R. Hilfer, Application of Fractional Calculus in Physics, World Science Publishing, Singapore, 2000.

[3] A. Nagih and G. Plateau, Fractional Problems: Overview of Applications and Solutions, Rairo-Recherche OperationnelleOper Press, New York, NY, USA, 1999.

[4] J.-P. Richard, "Time-delay systems: an overview of some recent advances and open problems," Automatica, vol. 39, no. 10, pp. 1667-1694, 2003.

[5] H. Ye, J. Gao, and Y. Ding, "A generalized Gronwall inequality and its application to a fractional differential equation," Journal of Mathematical Analysis and Applications, vol. 328, no. 2, pp. 1075-1081, 2007.

[6] M. Lazarevic, "Stability and stabilization of fractional order time delay systems," Scientific Technical Review, vol. 61, pp. 31-45, 2011.

[7] Y. Li, Y. Q. Chen, and I. Podlubny, "Stability of fractionalorder nonlinear dynamic systems: Lyapunov direct method and generalized Mittag-Leffler stability," Computers \& Mathematics with Applications, vol. 59, no. 5, pp. 1810-1821, 2010.

[8] D. Baleanu, A. Ranjba, S. Sadati et al., "Lyapunov-Krasovskii stability theorem for fractional systems with delay," Romanian Journal of Physics, vol. 56, pp. 636-643, 2011.

[9] S. Balochian, A. K. Sedigh, and A. Zare, "Stabilization of multiinput hybrid fractional-order systems with state delay," ISA Transactions, vol. 50, no. 1, pp. 21-27, 2011.

[10] M. Moze, J. Sabatier, and A. Oustaloup, "On bounded real lemma for fractional systems," in Proceedings of the 17th World Congress, International Federation of Automatic Control (IFAC '08), pp. 15267-15272, Seoul, Republic of Korea, July 2008.

[11] J. Zhuang and Y. Zhong, "State feedback $H_{\infty}$ optimal control for linear fractional-order systems," in Proceedings of the 29th Chinese Control Conference (CCC '10), pp. 1923-1927, Beijing, China, July 2010.

[12] J. Shen and J. Lam, "State feedback $H_{\infty}$ control of commensurate fractional-order systems," International Journal of Systems Science, vol. 45, no. 3, pp. 363-372, 2014.

[13] J.-H. She, M. Fang, Y. Ohyama, H. Hashimoto, and M. Wu, "Improving disturbance-rejection performance based on an equivalent-input-disturbance approach," IEEE Transactions on Industrial Electronics, vol. 55, no. 1, pp. 380-389, 2008.
[14] R.-J. Liu, M. Wu, G.-P. Liu, J. She, and C. Thomas, "Active disturbance rejection control based on an improved equivalent-inputdisturbance approach," IEEE/ASME Transactions on Mechatronics, vol. 18, no. 4, pp. 1410-1413, 2013.

[15] R.-J. Liu, G.-P. Liu, M. Wu, and Z.-Y. Nie, "Disturbance rejection for time-delay systems based on the equivalent-inputdisturbance approach," Journal of the Franklin Institute, vol. 351, no. 6, pp. 3364-3377, 2014.

[16] J. C. Trigeassou, N. Maamri, J. Sabatier, and A. Oustaloup, "A Lyapunov approach to the stability of fractional differential equations," Signal Processing, vol. 91, no. 3, pp. 437-445, 2011.

[17] Y.-H. Lan, H.-X. Huang, and Y. Zhou, "Observer-based robust control of $\alpha(1 \leq \alpha \leq 2)$ fractional-order uncertain systems: a linear matrix inequality approach," IET Control Theory \& Applications, vol. 6, no. 2, pp. 229-234, 2012.

[18] Y.-H. Lan and Y. Zhou, "Non-fragile observer-based robust control for a class of fractional-order nonlinear systems," Systems and Control Letters, vol. 62, no. 12, pp. 1143-1150, 2013.

[19] J.-B. Hu, G.-P. Lu, S.-B. Zhang, and L.-D. Zhao, "Lyapunov stability theorem about fractional system without and with delay," Communications in Nonlinear Science and Numerical Simulation, vol. 20, no. 3, pp. 905-913, 2015.

[20] O. Naifar, A. B. Makhlouf, and M. A. Hammami, "Comments on 'Lyapunov stability theorem about fractional system without and with delay,"' Communications in Nonlinear Science and Numerical Simulation, vol. 30, no. 1-3, pp. 360-361, 2016.

[21] D. W. C. Ho and G. Lu, "Robust stabilization for a class of discrete-time non-linear systems via output feedback: the unified LMI approach," International Journal of Control, vol. 76, no. 2, pp. 105-115, 2003.

[22] P. P. Khargonekar, I. R. Petersen, and K. Zhou, "Robust stabilization of uncertain linear systems: quadratic stabilizability and $H^{\infty}$ control theory," IEEE Transactions on Automatic Control, vol. 35, no. 3, pp. 356-361, 1990.

[23] A. Oustaloup, F. Levron, B. Mathieu, and F. M. Nanot, "Frequency-band complex noninteger differentiator: characterization and synthesis," IEEE Transactions on Circuits and Systems I: Fundamental Theory and Applications, vol. 47, no. 1, pp. 25-39, 2000. 


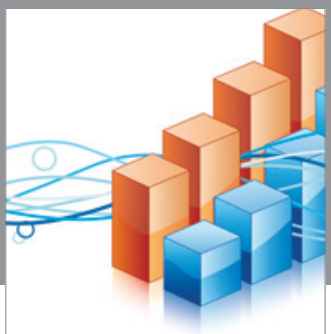

Advances in

Operations Research

vatem alat4

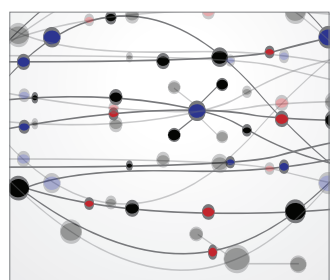

\section{The Scientific} World Journal
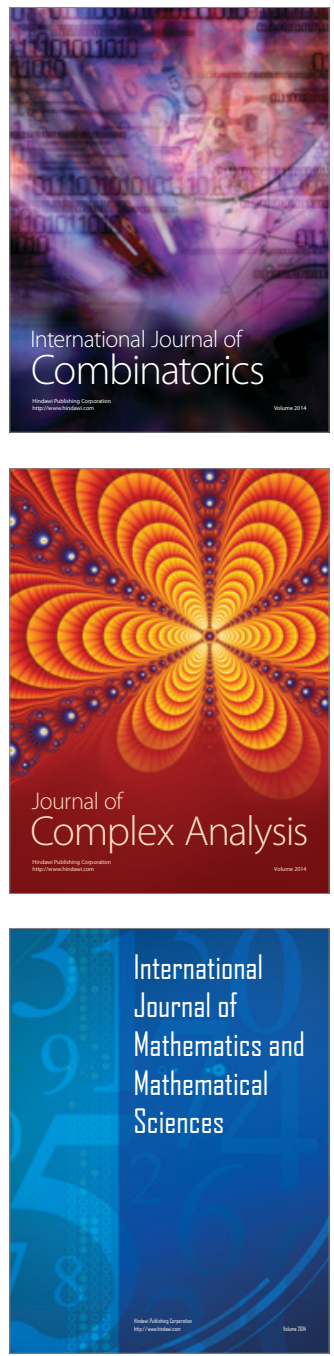
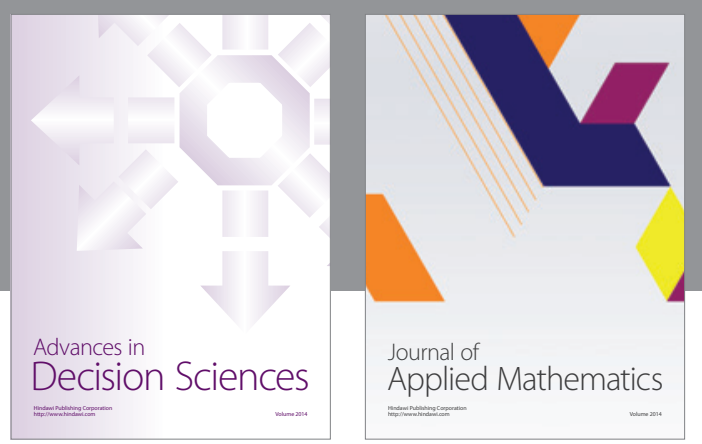

Algebra

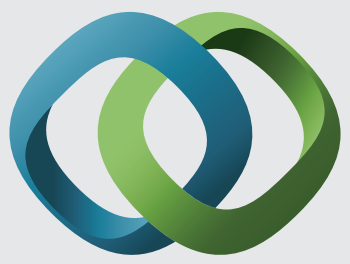

\section{Hindawi}

Submit your manuscripts at

http://www.hindawi.com
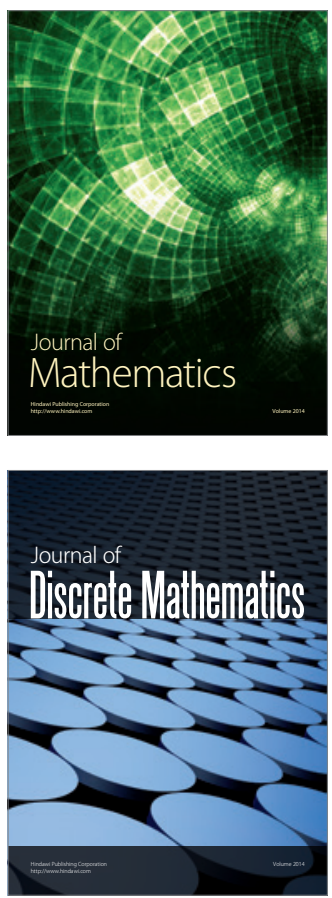

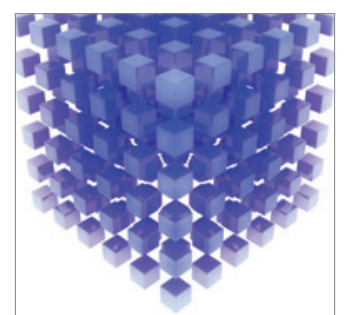

Mathematical Problems in Engineering
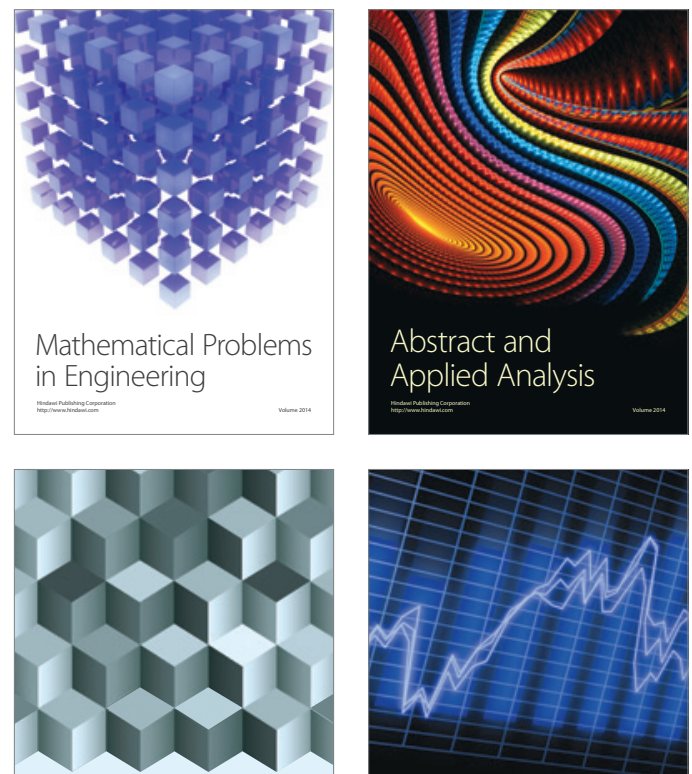

Journal of

Function Spaces

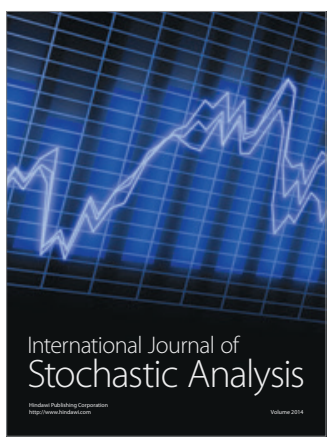

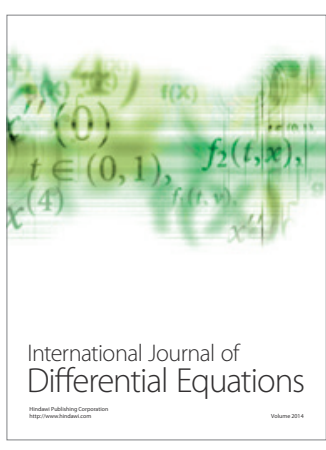
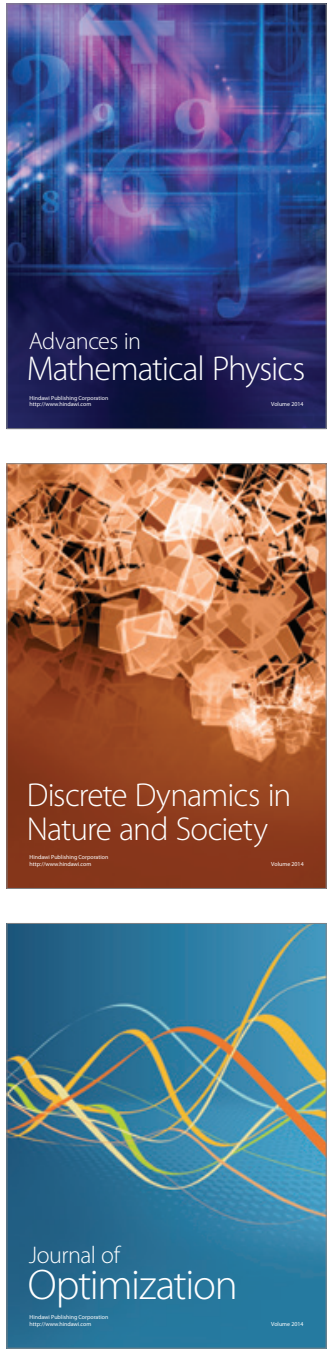